\title{
Numerical Study of Fisher's Equation by Finite Difference Schemes
}

\author{
Bader Saad Alshammari, Daoud Suleiman Mashat \\ Department of Mathematics, Numerical Analysis, King Abdulaziz University, Jeddah, Saudi Arabia \\ Email: bader--saad@hotmail.com,dmashat@kau.edu.sa
}

How to cite this paper: Alshammari, B.S. and Mashat, D.S. (2017) Numerical Study of Fisher's Equation by Finite Difference Schemes. Applied Mathematics, 8, 11001116.

https://doi.org/10.4236/am.2017.88083

Received: July 15, 2017

Accepted: August 15, 2017

Published: August 18, 2017

Copyright $\odot 2017$ by authors and Scientific Research Publishing Inc. This work is licensed under the Creative Commons Attribution International License (CC BY 4.0).

http://creativecommons.org/licenses/by/4.0/ (c) (i) Open Access

\begin{abstract}
This research paper represents a numerical approximation to three interesting equations of Fisher, which are linear, non-linear and coupled linear one dimensional reaction diffusion equations from population genetics. We studied accuracy in term of $L_{\infty}$ error norm by random selected grids along time levels for comparison with exact results. The test example demonstrates the accuracy, efficiency and versatility of the proposed schemes. It is shown that the numerical schemes give better solutions. Moreover, the schemes can be easily applied to a wide class of higher dimension non-linear reaction diffusion equations.
\end{abstract}

\section{Keywords}

Forward in Time and Centre in Space (FTCS), Taylor's Series, Crank Nicolson, Douglas Scheme

\section{Introduction}

Reaction diffusion equations arise as models for the densities of substances or organisms that disperse through space by Brownian motion, random walks, hydrodynamic turbulence, or similar mechanisms, and that react to each other and their surroundings in ways that affect their local densities [1]. Reaction diffusion models are in themselves deterministic, but they can be derived as limits of stochastic processes under suitable scaling. Specifically, they provide a modelling approach that allows us to translate assumptions about stochastic local movement into deterministic descriptions of global densities [1] [2]. Reaction diffusion models are spatially explicit, describe population densities, and treat space and time as continuous [1] [2] [3]. There are three major types of ecological phenomena that are supported by reaction diffusion equations: the existence of a minimal patch size necessary to support a population, the presence 
of travelling wave fronts corresponding to biological invasions, and the formation of spatial patterns [1] [2] [3].

\subsection{Diffusion}

Diffusion is a description of movement that arises as a result of an object or organism making many short movements in random directions. The diffusive description of random motion emerges as a continuum limit of such random walks when the length $\Delta x$ of each step and the time $\Delta t$ required for each step go to zero in such a way that the ratio $(\Delta x)^{2} / \Delta t$ remains constant. To understand how this works it is useful to consider a simple example in one space dimension. Suppose that an organism moves along a line by moving a distance $\Delta x$ to the left with probability $1 / 2$ or a distance $\Delta x$ to the right with probability $1 / 2$ at each time step $\Delta t$. Suppose that $\rho(x, t)$ is the probability that the organism is at location $x$ at time $t$. To arrive at that point at that time it must have been either one step to the left at time $t-\Delta t$ and then moved to the right, or one step to the right and have moved to the left. Thus, we have

$$
\rho(x, t)=\frac{1}{2} \rho(x+\Delta x, t-\Delta t)+\frac{1}{2} \rho(x-\Delta x, t-\Delta t)
$$

If we subtract $\rho(x, t-\Delta t)$ from both sides and divide by $\Delta t$ in Equation (1), we obtain,

$$
\begin{aligned}
& \frac{\rho(x, t)-\rho(x, t-\Delta t)}{\Delta t} \\
& =\frac{1}{2 \Delta t}[\rho(x+\Delta x, t-\Delta t)-2 \rho(x, t-\Delta t)+\rho(x-\Delta x, t-\Delta t)]
\end{aligned}
$$

Suppose that we now impose the diffusive scaling, $(\Delta x)^{2} / \Delta t=2 D$. Let us look at Equation (2),

$$
\begin{aligned}
& \frac{\rho(x, t)-\rho(x, t-\Delta t)}{\Delta t} \\
& =\frac{D}{(\Delta x)^{2}}[\rho(x+\Delta x, t-\Delta t)-2 \rho(x, t-\Delta t)+\rho(x-\Delta x, t-\Delta t)]
\end{aligned}
$$

From above Equation (3), the expression on the left is a difference quotient in $t$ and also the expression on the right is a second difference in $x$. Taking the limit of expression in Equation (3), as $(\Delta x, \Delta t) \rightarrow 0$, while in Equation (2) remains in force yields the diffusion equation,

$$
\frac{\partial \rho}{\partial t}=D \frac{\partial^{2} \rho}{\partial x^{2}}
$$

Mathematically this is identical to the heat equation. Note that the scaling, where $D$ is the square of the distance $\Delta x$ moved by the organism in a time unit $\Delta t$, produces a coefficient in front of the term $\partial^{2} \rho / \partial x^{2}$, which is equal to $1 / 2$ of the square of the distance moved per unit time. This interpretation of the diffusion coefficient $D$ is valid in any number of dimensions. 


\subsection{Reaction}

In the context of ecological models, the reaction terms in reaction diffusion equations and systems are typically the same as those that are used in nonspatial population models based on ordinary differential equations. Thus, for a single population, the reaction terms would be those that might occur in a model for a population density $\rho(t)$ of the form

$$
\frac{\partial \rho}{\partial t}=f(\rho)
$$

where $f(\rho)$ often has the form $f(\rho)=g(\rho) \rho$. Common choices for $f(\rho)$ are $f(\rho)=R \rho$ (linear growth), $f(\rho)=R \rho(1-\rho / K) \quad$ (logistic growth), or $f(\rho)=R \rho(\rho-a)(1-\rho / K)$ with $a \in(0, K)$ (growth with Allee effect). For systems, typical reaction terms are those that occur in non-spatial models for competition, mutualism, or predator-prey interactions. Those include Lotka-Volterra models, but also more general models such as predator-prey models with a functional response. In the case of systems the stability analysis often involves the eigenvalues of matrices obtained by linearising about the equilibria. Equilibria and eigenvalues play a similar role in the analysis of reaction diffusion models, but the eigenvalues generally are associated with differential operators rather than matrices.

Here is the outline of the article. In Section 2, we mentioned literature review according to scope of the equation and numerical treatment also in section 3, we derived governing equation and its three interesting types and in section 4, methodology is explained. In the section 5 , we discussed results in detail.

\section{Literature Review}

A well known researchers have studied such model problem, for example, Abdullaev [4] has studied the stability of symmetric travelling waves in the Cauchy problem for a more general case, also Logan has studied this problem using a perturbation method and found an approximate solution by expanding the solution in terms of a power series and in terms of some small parameters [5], whereas numerical solution found by Gazdag and Canosa [6] [7] which exhibits consistency with partial differential equations along initial and boundary condition. Both numerical schemes intimated in [7] are totally complicated and source of unexpected high frequency oscillations, which must be refine at each time step. Tang and Weber [8] [9] have studied Fisher's equation, using the Petrov-Galerkin method and Al-Khaled [10] has used the sinc collocation method to solve such model equation.

Recently, numerical solution to Fisher's equation, have studied by many researchers, such as Wang [11] give idea of exact solution and explicit solitary wave solutions which are associated with generalized form, also Dag [12] found solution of Fisher's equation numerically, using B-spline Galerkin method, whereas Qio and Sloan [13] built up numerical solutions of Fisher's equation by moving mesh method, meanwhile Ting [13] studied to solve generalized Fisher's 
equation by element free Galerkin method. Also modified cubic B-spline collocation method is used by Mittal and Jain [13] to study numerical solutions of non-linear Fisher's equation. Fisher's equation is studied numerically by Chandraker [13], also Tomasiello studied numerical stability of differential quadrature solutions of wave problems. Korkmaz and Dag applied polynomial differential quadrature method to study numerical solutions of non-linear Burger's equation [13]. Finite difference based methods have been applied by Kaysar [14] to solve Burger's and Fisher's equations numerically.

\section{Governing Equation}

In 1937 Fisher [15] and Kolmogorov et al. [16] investigated independently the Fisher Kolmogorov Petrovsky Piscounov (Fisher-KPP) equation, after that it is widely known as Fisher equation. This equation has many applications in science and engineering fields [6] [17]. The researchers studied some meaningful generalization of this equation, here we considered one generalization of this equation which is called as one component reaction diffusion equation. Many reaction diffusion equations have travelling wave fronts which play an important role in the understanding of physical, chemical, and biological phenomena [18]. Reaction-diffusion systems are mathematical models which explains how the concentration of one or more substances distributed in space changes under the influence of two processes, first one is local chemical reactions in which the substances are transformed into each other and second is the diffusion which causes the substances to spread out over a surface in space. Reaction-diffusion systems are naturally applied in chemistry. However, the system can also describe the dynamical processes of non-chemical nature. In this paper, we introduce the following three major Fisher's equations, which can be explained as.

\subsection{Linear Form of Fisher's Equation}

The linear form of Fisher's equation is as follows,

$$
u_{t}=\boldsymbol{\beta} u_{x x}+\boldsymbol{\alpha}(1-u(x, t)),
$$

where $\boldsymbol{\beta}$ is diffusive constant with value $0 \leq \boldsymbol{\beta} \leq 1$ and $\boldsymbol{\alpha}$ is reactive constant with value $0 \leq \boldsymbol{\alpha} \leq 1$. Also analytical solution to above Equation (6) is,

$$
u(x, t)=1-\frac{\cosh x}{\cosh 1}-\frac{16}{\pi^{2}} \sum_{n=1}^{\infty} \frac{(-1)^{n} \cos (2 n-1) \pi x / 2}{(2 n-1)\left[(2 n-1)^{2} \pi^{2}+4\right]} \mathrm{e}^{-\left(1+(2 n-1)^{2} \pi^{2} / 4\right) t},
$$

with boundary conditions are

$$
u(-1, t)=u(1, t)=0,
$$

and initial condition also,

$$
u(x, 0)=0 .
$$

\subsection{Coupled Linear System}

The coupled linear system is as follows, 


$$
\left.\begin{array}{l}
u_{t}=u_{x x}+(u(x, t)-v(x, t))+F(x, t) \\
v_{t}=v_{x x}+(u(x, t)+v(x, t))+G(x, t)
\end{array}\right\}
$$

with analytical solution,

$$
\left.\begin{array}{l}
u(x, t)=\mathrm{e}^{t} \sin x \\
v(x, t)=\mathrm{e}^{t} \cos x
\end{array}\right\}
$$

\subsection{Nonlinear Generalized Fisher's Equation}

The generalized form of nonlinear Fisher's equation is as follows,

$$
u_{t}=u_{x x}+u(1-u)\left(u-\alpha_{1}\right), \quad 0<\alpha_{1}<1
$$

with analytical solution,

$$
u(x, t)=\frac{1}{2}\left(1+\alpha_{1}\right)+\left(\frac{1}{2}-\frac{1}{2} \alpha_{1}\right) \tanh \left[\sqrt{2}\left(1-\alpha_{1}\right) \frac{x}{4}+\frac{\left(1-\alpha_{1}^{2}\right)}{4} t\right],
$$

the initial and boundary conditions are taken from the exact solution (13).

\section{Numerical Methods}

Let us apply numerical methods like Finite Difference Schemes (Forward in time and central in space (FTCS), Crank Nicolson (CN) and Douglas), to solve such Equations ((6), (10), (12)) in finite domain $\Omega=[0,1]$. We partitioned the interval $[a, b]$ into $\mathrm{n}$ equal parts of width $\mathrm{h}$. Place a grid on the rectangle region $\mathrm{R}$ by drawing vertical and horizontal lines through the points with coordinates $x_{i}$, where $x_{i}=a+i h$ for each $i=0,1,2, \cdots, n$ also the lines $x=x_{i}$ represent grid lines, we assume $t_{n}=n t, n=0,1, \cdots$ where $n$ is the time grid step size. We denote the exact and numerical solutions at the grid point $\left(x_{m}, t_{n}\right)$ by $U_{m}^{n}$ and $u_{m}^{n}$ respectively.

\subsection{Forward in Time and Center in Space (FTCS) Scheme}

We consider forward in time and center in space (FTCS) explicit scheme by substituting the forward difference approximation for the time derivative and the central difference approximation for the space derivative in Equations ((6), (10), (12)) respectively, we get the following

$$
u_{i}^{n+1}=u_{i}^{n}+\mathbf{R}\left(u_{i+1}^{n}-2 u_{i}^{n}+u_{i-1}^{n}\right)+\mathbf{Q}\left(1-u_{i}^{n}\right),
$$

where $\mathbf{R}=\frac{k \beta}{h^{2}}$, and $\mathbf{Q}=k \alpha$. Above equation (14) represents descritezation to linear form of Fisher's equation.

$$
\left.\begin{array}{l}
u_{i}^{n+1}=u_{i}^{n}+\mathbf{R}\left(u_{i+1}^{n}-2 u_{i}^{n}+u_{i-1}^{n}\right)+k u_{i}^{n}-k v_{i}^{n}+k F\left(u_{i}^{n}, v_{i}^{n}, x, t\right) \\
v_{i}^{n+1}=v_{i}^{n}+\mathbf{R}\left(v_{i+1}^{n}-2 v_{i}^{n}+v_{i-1}^{n}\right)+k u_{i}^{n}+k v_{i}^{n}+k G\left(u_{i}^{n}, v_{i}^{n}, x, t\right)
\end{array}\right\}
$$

above Equation (15) represents descritezation to coupled linear system.

$$
u_{i}^{n+1}=u_{i}^{n}+\mathbf{R}\left(u_{i+1}^{n}-2 u_{i}^{n}+u_{i-1}^{n}\right)+k u_{i}^{n}\left(1-u_{i}^{n}\right)\left(u_{i}^{n}-\alpha_{1}\right),
$$


above Equation (16) represents descritezation to nonlinear generalized form of Fisher's equation.

Since the one dimensional Fisher's equation or system is well posed, make sure the spacing $h$ for spatial and $k$ for time of the finite difference grid are made sufficiently small [6] [17] [19]. The FTCS scheme, from Equations (14)-(16), is classified as explicit because the value of $u_{i}^{n+1}$ at the $(n+1)$ th time level may be calculated directly from known value of $u_{i}^{n}$ at previous time levels. It is a two level method because values of $u(x, t)$ at only two levels of time are involved in the approximating finite difference equation [6] [17] [19]. There is no best method for obtaining approximating difference formula, the only requirement is that the formula, having been obtained, must pass certain tests of accuracy, consistency, stability and convergence [20] [21] [22]. By Von Neumann stability analysis, the FTCS scheme is always conditionally stable, which is $0<\mathbf{R} \leq \frac{1}{2}$.

\subsection{Crank Nicolson Implicit Scheme}

Let us apply implicit finite difference scheme, which is Crank Nicolson. This method uses central finite difference approximation for both time and space derivatives at the point $\left(x_{m}, t_{n}\right)$ [6]. For diffusion equations (and many other equations), it can be shown that, the Crank Nicolson method is unconditionally stable [23] [24] [25]. However, the approximate solutions can still contain (decaying) spurious oscillations if the ratio of time step $k$ times to the square of space step $h^{2}$, is large (typically larger than $1 / 2$ per Von-Neumann stability analysis) [21] [26] [27]. For this reason, whenever large time steps or high spatial resolution is necessary, the less accurate backward Euler method is often used, which is both stable and immune to oscillations [24] [25] [27] [28] [29] [30]. In this method, we consider the Equations ((6), (10), (12)) respectively, in the following way,

$$
\begin{aligned}
\frac{u_{i}^{n+1}-u_{i}^{n}}{k}= & \frac{k \beta}{2 h^{2}} \delta_{x}^{2}\left[u_{i}^{n+1}+u_{i}^{n}\right]+\alpha\left(1-\frac{1}{2}\left(u_{i}^{n+1}+u_{i}^{n}\right)\right) \\
& -\mathbf{R}_{1} u_{i+1}^{n+1}+\left(1+2 \mathbf{R}_{1}+0.5 \mathbf{Q}_{1}\right) u_{i}^{n+1}-\mathbf{R}_{1} u_{i-1}^{n+1} \\
= & \mathbf{R}_{1} u_{i+1}^{n}+\left(1-2 \mathbf{R}_{1}-0.5 \mathbf{Q}_{1}\right) u_{i}^{n}+\mathbf{R}_{1} u_{i-1}^{n}+\mathbf{Q}_{1}
\end{aligned}
$$

where $\mathbf{R}_{1}=\frac{k \beta}{2 h^{2}}$ and $\mathbf{Q}_{1}=k \alpha$. Above Equation (17) represents descritezation using Crank Nicolson to linear form of Fisher's equation. Now let us look at coupled linear system, in the following way,

$$
\left.\begin{array}{l}
-\mathbf{R}_{1} u_{i+1}^{n+1}+\left(1+2 \mathbf{R}_{1}-0.5 k\right) u_{i}^{n+1}+0.5 k v_{i}^{n+1}-\mathbf{R}_{1} u_{i-1}^{n+1} \\
=\mathbf{R}_{1} u_{i+1}^{n}+\left(1-2 \mathbf{R}_{1}+0.5 k\right) u_{i}^{n}-0.5 k v_{i}^{n}+k F\left(\frac{1}{2}\left(u_{i}^{n+1}+u_{i}^{n}\right), \frac{1}{2}\left(v_{i}^{n+1}+v_{i}^{n}\right), x, t\right) \\
-\mathbf{R}_{1} v_{i+1}^{n+1}+\left(1+2 \mathbf{R}_{1}-0.5 k\right) v_{i}^{n+1}-0.5 k u_{i}^{n+1}-\mathbf{R}_{1} v_{i-1}^{n+1} \\
=\mathbf{R}_{1} v_{i+1}^{n}+\left(1-2 \mathbf{R}_{1}+0.5 k\right) v_{i}^{n}+0.5 k u_{i}^{n}+k G\left(\frac{1}{2}\left(u_{i}^{n+1}+u_{i}^{n}\right), \frac{1}{2}\left(v_{i}^{n+1}+v_{i}^{n}\right), x, t\right)
\end{array}\right\}
$$


Above equation (18) represents descritezation using Crank Nicolson to coupled linear system. Now let us look at generalized nonlinear Fisher's equation using Crank Nicolson,

$$
\begin{aligned}
& -\mathbf{R}_{1} u_{i+1}^{n+1}+\left(1+2 \mathbf{R}_{1}\right) u_{i}^{n+1}-\mathbf{R}_{1} u_{i-1}^{n+1} \\
& =\mathbf{R}_{1} u_{i+1}^{n}+\left(1-2 \mathbf{R}_{1}\right) u_{i}^{n}+\frac{k}{2}\left(u_{i}^{n+1}+u_{i}^{n}\right)\left(1-0.5\left(u_{i}^{n+1}+u_{i}^{n}\right)\right)\left(0.5\left(u_{i}^{n+1}+u_{i}^{n}\right)-\alpha_{1}\right)
\end{aligned}
$$

\subsection{Fourth Order Accurate Implicit Scheme}

Let us apply another implicit scheme to Equations ((6), (12)) in an order respectively.

$$
\left.\begin{array}{l}
\frac{u_{i}^{n+1}-u_{i}^{n}}{k}=\frac{\beta}{2 h^{2}}\left[1+\delta_{x}^{2}\right]^{-1}\left(u_{i}^{n+1}+u_{i}^{n}\right)+\alpha\left(1-\frac{1}{2}\left(u_{i}^{n+1}+u_{i}^{n}\right)\right) \\
{\left[1+\delta_{x}^{2}\right]\left(\frac{u_{i}^{n+1}-u_{i}^{n}}{k}\right)=\frac{\beta}{2 h^{2}}\left(u_{i}^{n+1}+u_{i}^{n}\right)+\left[1+\delta_{x}^{2}\right] \alpha\left(1-\frac{1}{2}\left(u_{i}^{n+1}+u_{i}^{n}\right)\right)} \\
\left(u_{i}^{n+1}-u_{i}^{n}\right)+\delta_{x}^{2}\left(u_{i}^{n+1}-u_{i}^{n}\right)=\mathbf{R}_{1}\left(u_{i}^{n+1}+u_{i}^{n}\right)+\left[1+\delta_{x}^{2}\right] \alpha\left(1-\frac{1}{2}\left(u_{i}^{n+1}+u_{i}^{n}\right)\right)
\end{array}\right\}
$$

Now Douglas scheme to nonlinear generalized Fisher's equation,

$$
\left.\begin{array}{l}
\frac{u_{i}^{n+1}-u_{i}^{n}}{k}=\frac{\beta}{2 h^{2}}\left[1+\delta_{x}^{2}\right]^{-1}\left(u_{i}^{n+1}+u_{i}^{n}\right)+\frac{1}{2}\left(u_{i}^{n+1}+u_{i}^{n}\right)\left(1-\frac{1}{2}\left(u_{i}^{n+1}+u_{i}^{n}\right)\right)\left(\frac{1}{2}\left(u_{i}^{n+1}+u_{i}^{n}\right)-\alpha_{1}\right) \\
{\left[1+\delta_{x}^{2}\right]\left(\frac{u_{i}^{n+1}-u_{i}^{n}}{k}\right)=\frac{\beta}{2 h^{2}}\left(u_{i}^{n+1}+u_{i}^{n}\right)+\left[1+\delta_{x}^{2}\right] \frac{1}{2}\left(u_{i}^{n+1}+u_{i}^{n}\right)\left(1-\frac{1}{2}\left(u_{i}^{n+1}+u_{i}^{n}\right)\right)\left(\frac{1}{2}\left(u_{i}^{n+1}+u_{i}^{n}\right)-\alpha_{1}\right)} \\
{\left[1+\delta_{x}^{2}\right]\left(u_{i}^{n+1}-u_{i}^{n}\right)=\mathbf{R}_{1}\left(u_{i}^{n+1}+u_{i}^{n}\right)+\left[1+\delta_{x}^{2}\right] \frac{1}{2}\left(u_{i}^{n+1}+u_{i}^{n}\right)\left(1-\frac{1}{2}\left(u_{i}^{n+1}+u_{i}^{n}\right)\right)\left(\frac{1}{2}\left(u_{i}^{n+1}+u_{i}^{n}\right)-\alpha_{1}\right)}
\end{array}\right\}
$$

\section{Error Norms}

The aim of the accuracy is assessed by some redefined norms, associated with the consistency of the finite difference schemes, such scaled measurement to error defined in term of norms specially $L_{\infty}$, which is outlined below:

$$
L_{\infty}=\max _{i}\left|u_{i}^{\text {Exact }}-u_{i}^{\text {Approximation }}\right|
$$

\section{Results}

Numerical computations have been performed using the uniform grid. We used FTCS, Crank Nicolson and Douglas finite difference schemes to analyse numerical behaviour of simple linear Fisher;s equation, one dimensional linear coupled system and non-linear Fisher's equation respectively. First we look at the linear Fisher's equation by finite difference schemes as in Table 1, we used FTCS explicit scheme with some variations in grid size and $h$ (space step) is changed according to the grid sizes. this table explains the second order accuracy in term of $L_{\infty}$ norm, of the explicit numerical scheme also Table 2 explains results for FTCS with different time steps $(k)$. As we know that FTCS scheme is always conditionally stable and we can see from Table 2. In Table 3, we used implicit Crank Nicolson finite difference scheme, which shows that results with 
$k=0.0001, t=1$, interval $=[-1,1]$ and different grid sizes with $h$ changes accordingly also Table 4 explains the method with different $k$ (time steps). Table 5 shows results using Douglas scheme with $k=0.0001, t=1$, interval $=[-1,1]$ and different grid sizes with $h$ changes accordingly also Table 6 shows results using Douglas scheme with Grid $=71 \times 71, t=1$, interval $=[-1,1]$ and different $k$. In Table 7 we represent results for linear Fisher's equation with comparison of two implicit schemes and Douglas improves and encourages our solution.

Secondly, we look at the coupled linear system by finite difference schemes as in Table 8 and Table 9, we used FTCS explicit scheme with some variations in grid size and $h$ (space step) is changed according to the grid sizes. these tables explain the second order accuracy in term of $L_{\infty}$ norm and also classical simple error as Error $=|U(x, t)-u(x, t)|$, by using both explicit and implicit schemes.

Lastly, we look at the generalized Fisher's equation by finite difference schemes as in Table 10, we used FTCS explicit scheme with some variations in grid size and $h$ (space step) is changed according to the grid sizes. this table explains the second order accuracy in term of $L_{\infty}$ norm, of the explicit numerical scheme. Table 11 shows results for Crank Nicolson to generalized non-linear Fisher's equation along Table 12 shows Douglas results at different grid sizes.

Table 1. This table shows results using FTCS explicit scheme with $k=0.0001, t=1$, interval $=[-1,1]$ and different grid sizes with $h$ changes accordingly.

\begin{tabular}{cccc}
\hline Grids & $k=$ Time Step & $L_{\infty}$ & $h=$ Space Step \\
\hline $51 \times 51$ & 0.0001 & 0.115 & 0.0400 \\
$101 \times 101$ & 0.0001 & 0.0115 & 0.0200 \\
$225 \times 225$ & 0.0001 & Inf & 0.0089 \\
$1011 \times 1011$ & 0.0001 & Inf & 0.0020 \\
\hline
\end{tabular}

Table 2. This table shows results using FTCS explicit scheme with Grid $=71 \times 71, t=1$, interval $=[-1,1]$ and different $k$.

\begin{tabular}{ccc}
\hline$k=$ Time & $L_{\infty}$ & $t$ \\
\hline 0.01 & $6.6408 e^{+161}$ & 1 \\
0.001 & $\operatorname{Inf}$ & 1 \\
0.0001 & 0.0115 & 1 \\
\hline
\end{tabular}

Table 3. This table shows results using Crank Nicolson scheme with $k=0.0001, t=1$, interval $=[-1,1]$ and different grid sizes with $h$ changes accordingly.

\begin{tabular}{cccc}
\hline Grids & $k=$ Time Step & $L_{\infty}$ & $h=$ Space Step \\
\hline $51 \times 51$ & 0.0001 & $3.1810 e^{-5}$ & 0.0400 \\
$101 \times 101$ & 0.0001 & $7.9538 e^{-6}$ & 0.0200 \\
$225 \times 225$ & 0.0001 & $1.5852 e^{-6}$ & 0.0089 \\
$1011 \times 1011$ & 0.0001 & $7.7977 e^{-8}$ & 0.0020 \\
\hline
\end{tabular}


Table 4. This table shows results using Crank Nicolson scheme with Grid $=71 \times 71, t=1$, interval $=[-1,1]$ and different $k$.

\begin{tabular}{ccc}
\hline$k=$ Time & $L_{\infty}$ & $t$ \\
\hline 0.01 & $1.6232 e^{-5}$ & 1 \\
0.001 & $1.6231 e^{-5}$ & 1 \\
0.0001 & $1.6231 e^{-5}$ & 1 \\
\hline
\end{tabular}

Table 5. This table shows results using Douglas scheme with $k=0.0001, t=1$, interval $=[-1,1]$ and different grid sizes with $h$ changes accordingly.

\begin{tabular}{cccc}
\hline Grids & $k=$ Time Step & $L_{\infty}$ & $h=$ Space Step \\
\hline $51 \times 51$ & 0.0001 & $2.5452 e^{-9}$ & 0.0400 \\
$101 \times 101$ & 0.0001 & $1.5913 e^{-10}$ & 0.0200 \\
$225 \times 225$ & 0.0001 & $5.6298 e^{-12}$ & 0.0089 \\
$1011 \times 1011$ & 0.0001 & $4.4561 e^{-12}$ & 0.0020 \\
\hline
\end{tabular}

Table 6. This table shows results using Douglas scheme with Grid $=71 \times 71, t=1$, interval $=[-1,1]$ and different $k$.

\begin{tabular}{ccc}
\hline$k=$ Time & \multicolumn{1}{c}{$L_{\infty}$} & $t$ \\
\hline 0.01 & $6.6254 e^{-10}$ & 1 \\
0.001 & $6.6250 e^{-10}$ & 1 \\
0.0001 & $6.6248 e^{-10}$ & 1 \\
\hline
\end{tabular}

Table 7. This table shows comparison between Crank Nicolson and Douglas schemes with $k=0.0001, t=1$ and interval $=[-1,1]$.

\begin{tabular}{cccccc}
\hline \multicolumn{3}{c}{ Crank Nicolson } & \multicolumn{3}{c}{ Douglas } \\
\hline Grid & $L_{\infty}$ & $h=$ Space Step & Grid & $L_{\infty}$ & $h=$ Space Step \\
$31 \times 31$ & $8.8330 e^{-5}$ & 0.0667 & $31 \times 31$ & $1.9637 e^{-8}$ & 0.0667 \\
$45 \times 45$ & $4.1075 e^{-5}$ & 0.0455 & $45 \times 45$ & $1.9637 e^{-8}$ & 0.0455 \\
$77 \times 77$ & $1.3770 e^{-5}$ & 0.0263 & $77 \times 77$ & $4.7676 e^{-10}$ & 0.0263 \\
\hline
\end{tabular}

Table 8. This table shows results using FTCS explicit scheme with $k=0.0001, t=0.1$ and interval $=[-3,3]$.

\begin{tabular}{ccccccccc}
\hline Grid & $U_{\text {exact }}$ & $u_{\text {app. }}$ & Error $_{1}$ & $L_{\infty} 1$ & $V_{\text {exact }}$ & $V_{\text {app. }}$ & Error $_{2}$ & $L_{\infty} 2$ \\
\hline $51 \times 51$ & -0.41156459 & -0.375022428 & 0.0365 & 0.0981 & -1.02567897 & -0.93461058 & 0.0911 & 0.0981 \\
$71 \times 71$ & 0.77048108 & 0.706718997 & 0.0638 & 0.0914 & 0.79231412 & 0.72674521 & 0.0656 & 0.0915 \\
$151 \times 151$ & 0.98573361 & 0.947763396 & 0.0380 & 0.0426 & -0.49973194 & -0.48048240 & 0.0192 & 0.0426 \\
$201 \times 201$ & 0.94172500 & 0.946871908 & 0.0051 & 0.0060 & 0.57840883 & 0.58157006 & 0.0032 & 0.0060 \\
$271 \times 271$ & 0.37021916 & 0.402856751 & 0.0326 & 0.0974 & -1.04131673 & -1.13311659 & 0.0918 & 0.0974 \\
\hline
\end{tabular}


Table 9. This table shows results using Crank Nicolson implicit scheme with $k=0.00001, t=0.001$ and interval $=[-10,10]$.

\begin{tabular}{ccccccccc}
\hline Grid & $U_{\text {exact }}$ & $u_{\text {app. }}$ & Error $_{1}$ & $L_{\infty} 1$ & $V_{\text {exact }}$ & $v_{\text {app. }}$ & Error $_{2}$ & $L_{\infty} 2$ \\
\hline $51 \times 51$ & -0.99034809 & -0.9903612342 & $1.3 e^{-5}$ & $1.3 e^{-5}$ & -0.14564560 & -0.1456475382 & $1.9 e^{-6}$ & $1.3 e^{-5}$ \\
$71 \times 71$ & 0.910207179 & 0.91021335413 & $6.1 e^{-6}$ & $6.7 e^{-6}$ & -0.41656319 & -0.4165660175 & $2.8 e^{-6}$ & $6.7 e^{-6}$ \\
$151 \times 151$ & -0.79446192 & -0.7944631049 & $1.1 e^{-6}$ & $1.4 e^{-6}$ & 0.608959970 & 0.60896087175 & $9.0 e^{-7}$ & $1.4 e^{-6}$ \\
$201 \times 201$ & -0.84231287 & -0.8423135783 & $7.0 e^{-7}$ & $8.3 e^{-7}$ & 0.540842878 & 0.54084332896 & $4.5 e^{-7}$ & $8.3 e^{-7}$ \\
$271 \times 271$ & -0.09140859 & -0.0914086402 & $4.1 e^{-8}$ & $9.2 e^{-6}$ & -0.99681817 & -0.9968186284 & $4.5 e^{-7}$ & $1.4 e^{-5}$ \\
\hline
\end{tabular}

Table 10. This Table shows results using FTCS explicit scheme with $k=0.0001, t=0.1$, interval $=[-10,10], A=0.5$, and different grid sizes with $h$ changes accordingly.

\begin{tabular}{cccc}
\hline Grids & $k=$ Time Step & $L_{\infty}$ & $h=$ Space Step \\
\hline $21 \times 21$ & 0.0001 & 0.0047 & 1 \\
$61 \times 61$ & 0.0001 & 0.0047 & 0.3333 \\
$121 \times 121$ & 0.0001 & 0.0047 & 0.1667 \\
$301 \times 301$ & 0.0001 & 0.0047 & 0.0667 \\
\hline
\end{tabular}

Table 11. This Table shows results using Crank Nicolson scheme with $k=0.0001, t=0.1$, interval $=[-10,10], A=0.5$ and different grid sizes with $h$ changes accordingly.

\begin{tabular}{cccc}
\hline Grids & $k=$ Time Step & $L_{\infty}$ & $h=$ Space Step \\
\hline $21 \times 21$ & 0.0001 & $7.9231 e^{-6}$ & 1 \\
$61 \times 61$ & 0.0001 & $9.7270 e^{-7}$ & 0.3333 \\
$121 \times 121$ & 0.0001 & $2.9720 e^{-7}$ & 0.1667 \\
$301 \times 301$ & 0.0001 & $1.0864 e^{-7}$ & 0.0667 \\
\hline
\end{tabular}

Table 12. This Table shows results using Douglas scheme with $t=0.1$ and interval= $[-10,10]$.

\begin{tabular}{ccccc}
\hline$A$ & Grid & $L_{\infty}$ & $k=$ Time & $h=$ Space Step \\
\hline 0.1 & $41 \times 41$ & 0.0029 & 0.0001 & 0.5000 \\
0.4 & $60 \times 60$ & 0.0103 & 0.0001 & 0.3390 \\
0.6 & $131 \times 131$ & 0.0079 & 0.0001 & 0.1538 \\
\hline
\end{tabular}

To analyse the graphic representation to linear Fisher's equation, we have Figure 1, by FTCS along Figures 2-4 by Crank Nicolson and by Douglas, Figure 5 enhance our knowledge. Figure 6 \& Figure 7 show results for coupled linear system by FTCS and Crank Nicolson. Also Figure 8 \& Figure 9 show results for non-linear generalized Fisher's equation by Crank Nicolson and Douglas schemes respectively.

\section{Conclusion}

In this paper, the solution to linear form of the Fishers equation, coupled linear 
system and generalized Fisher's equation is successfully approximated by a various numerical finite difference schemes. Two of them are implicit in nature such as Crank Nicolson and Douglas and one is explicit FTCS schemes. We have to pay attention to parameter $\mathbf{R}$, which can stabilize the results as we can see from figures and tables. For instant, Von-Neumann's method of stability analysis can not be used other than locally, since it only applies to linear finite
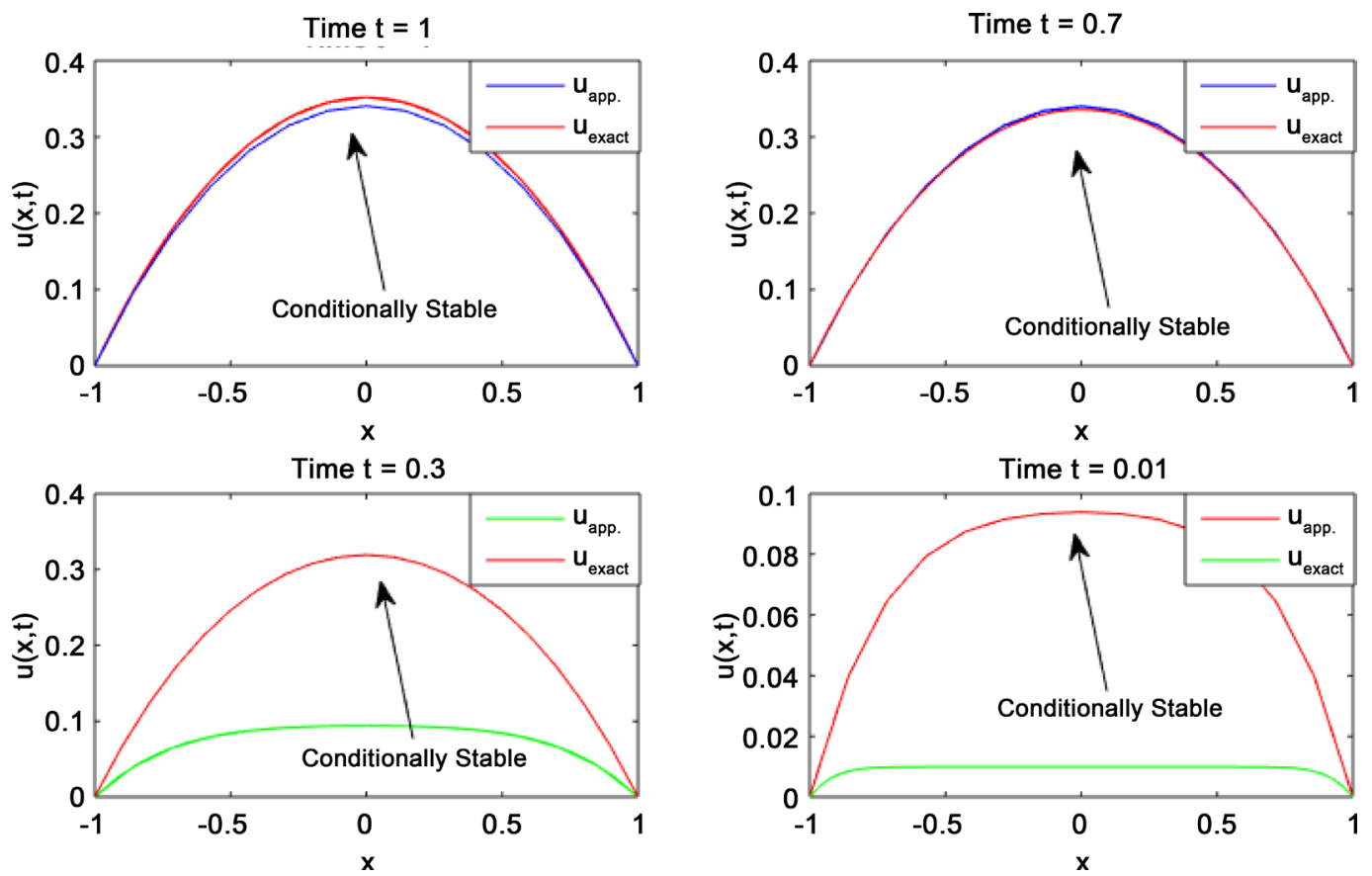

Figure 1. Results for different time level, with fixed grid size and time step, using FTCS scheme.
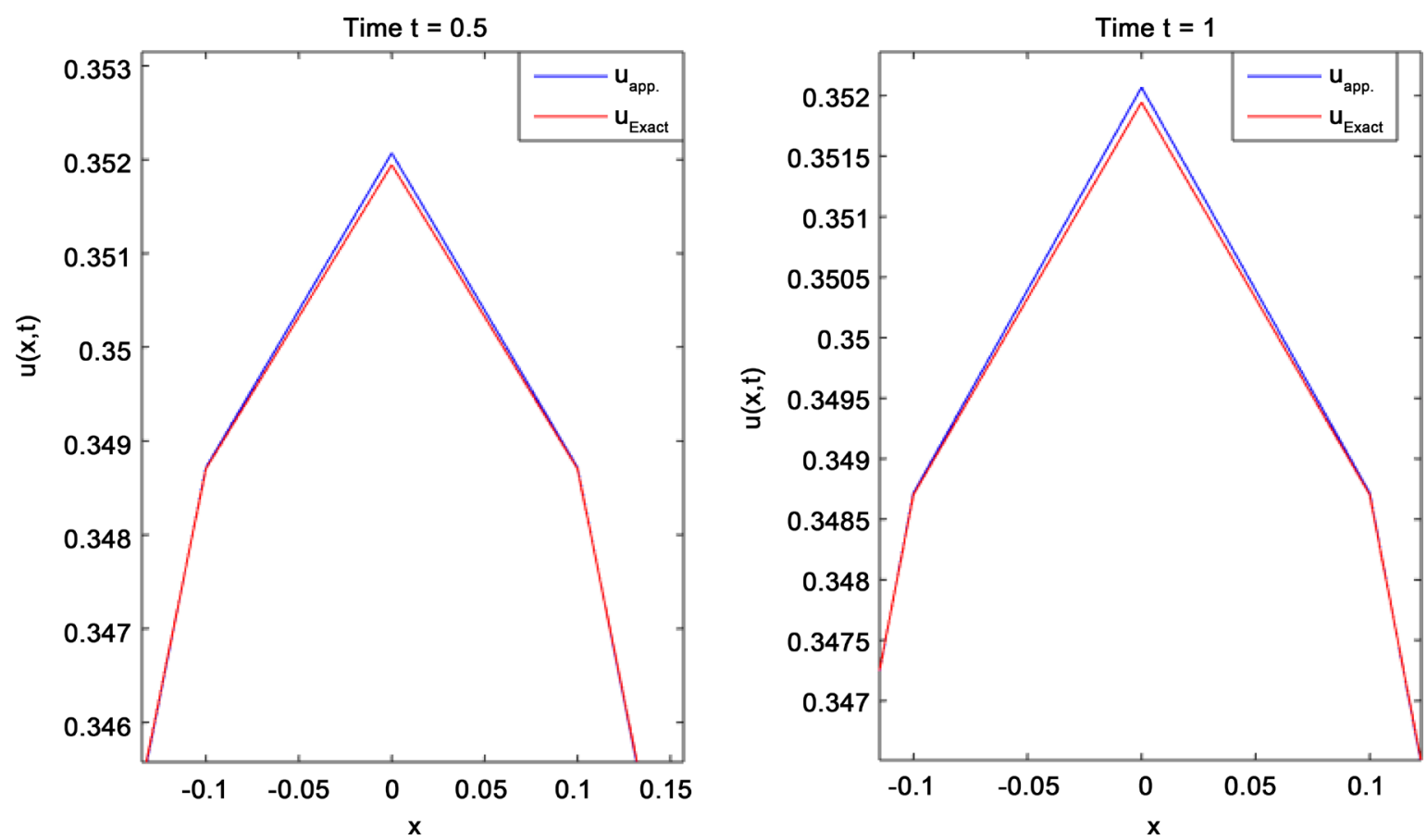

Figure 2. Results for different time level, with fixed grid size and time step, using Crank Nicolson scheme after zoom out. 

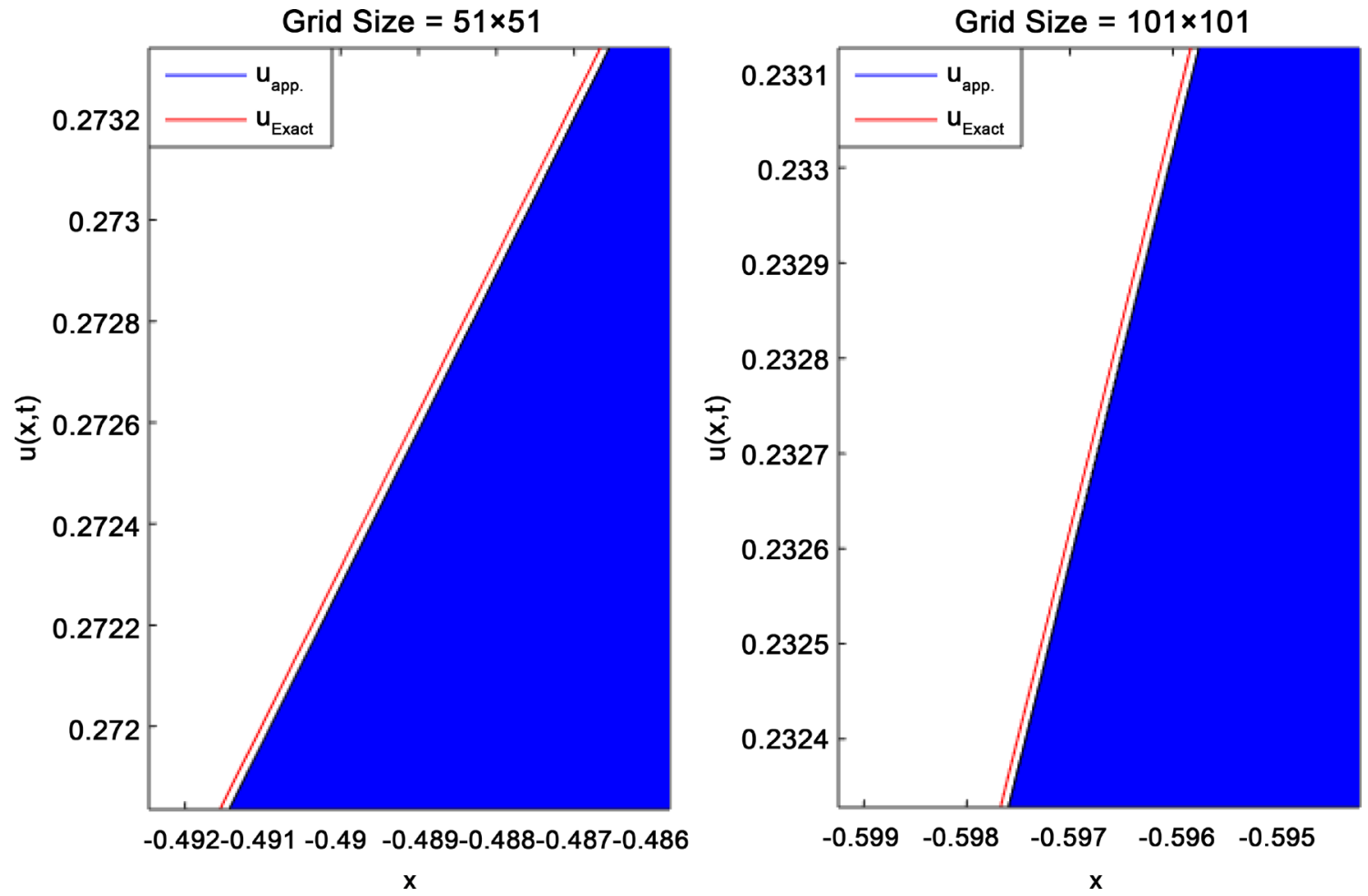

Figure 3. Results for different grid sizes with time step, and find common region of interaction, using Crank Nicolson scheme.
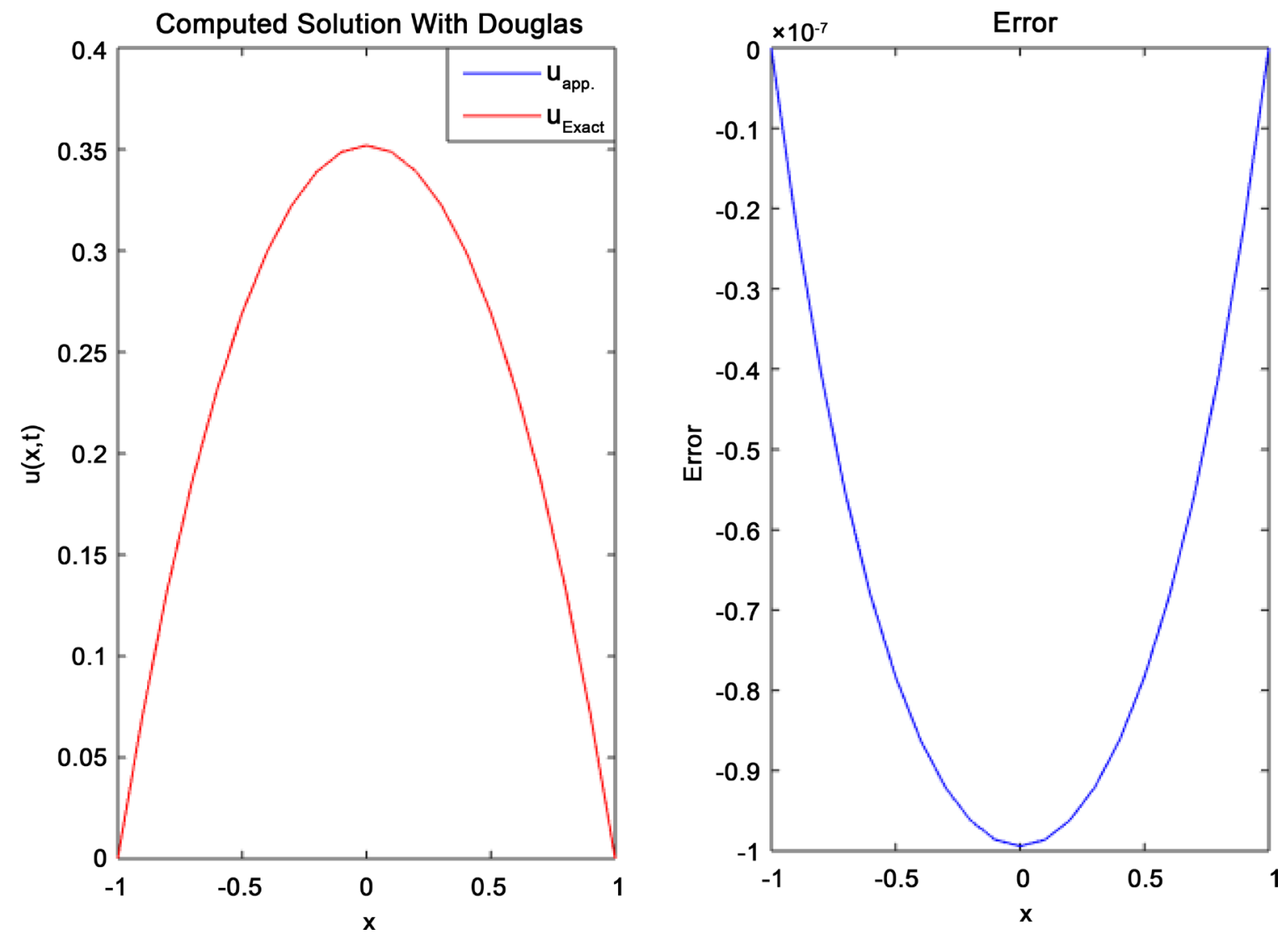

Figure 4. Results for Douglas scheme and error profile. 

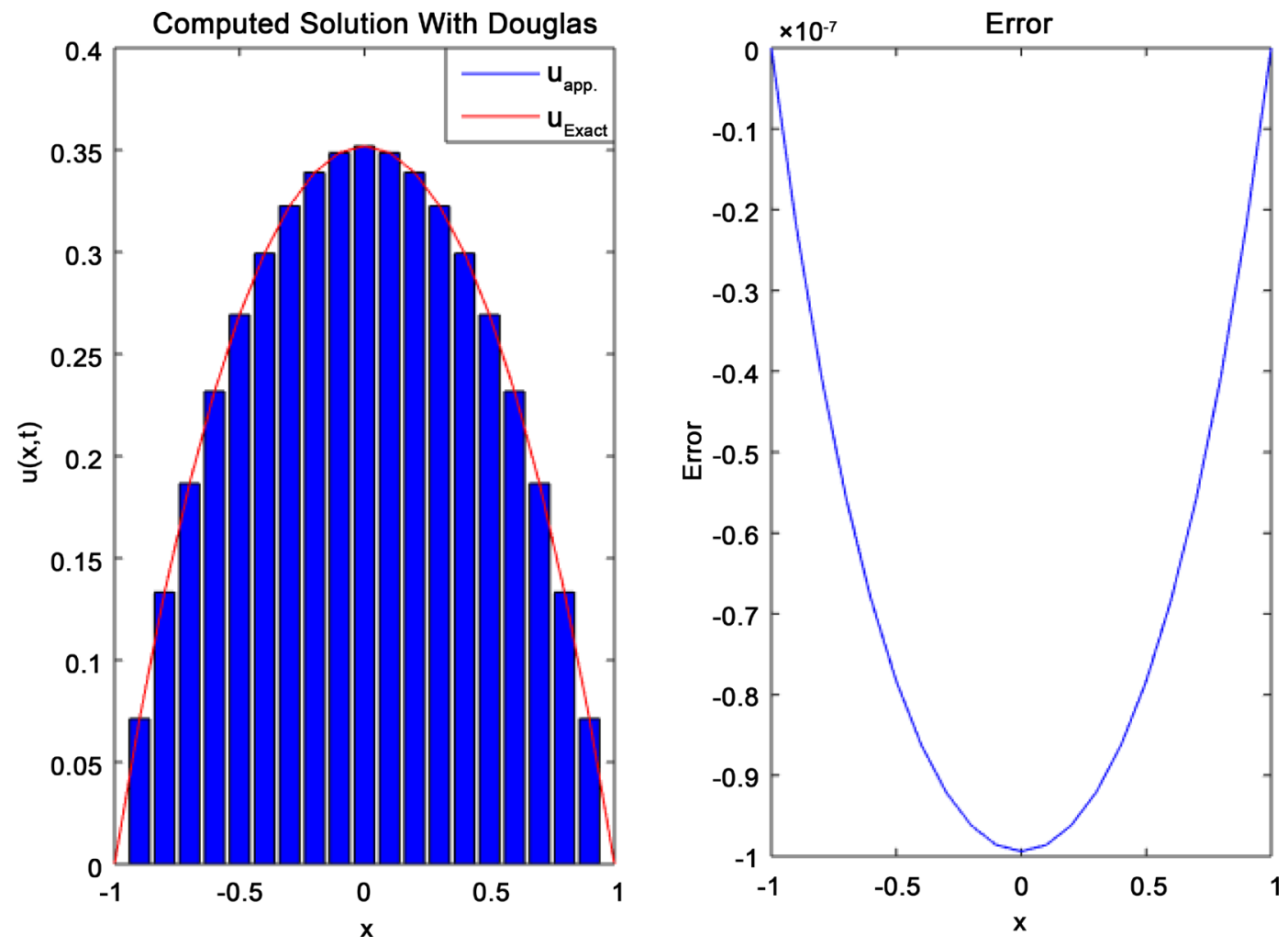

Figure 5. Results for Douglas scheme and error profile with common region.

Computed Solution With FTCS
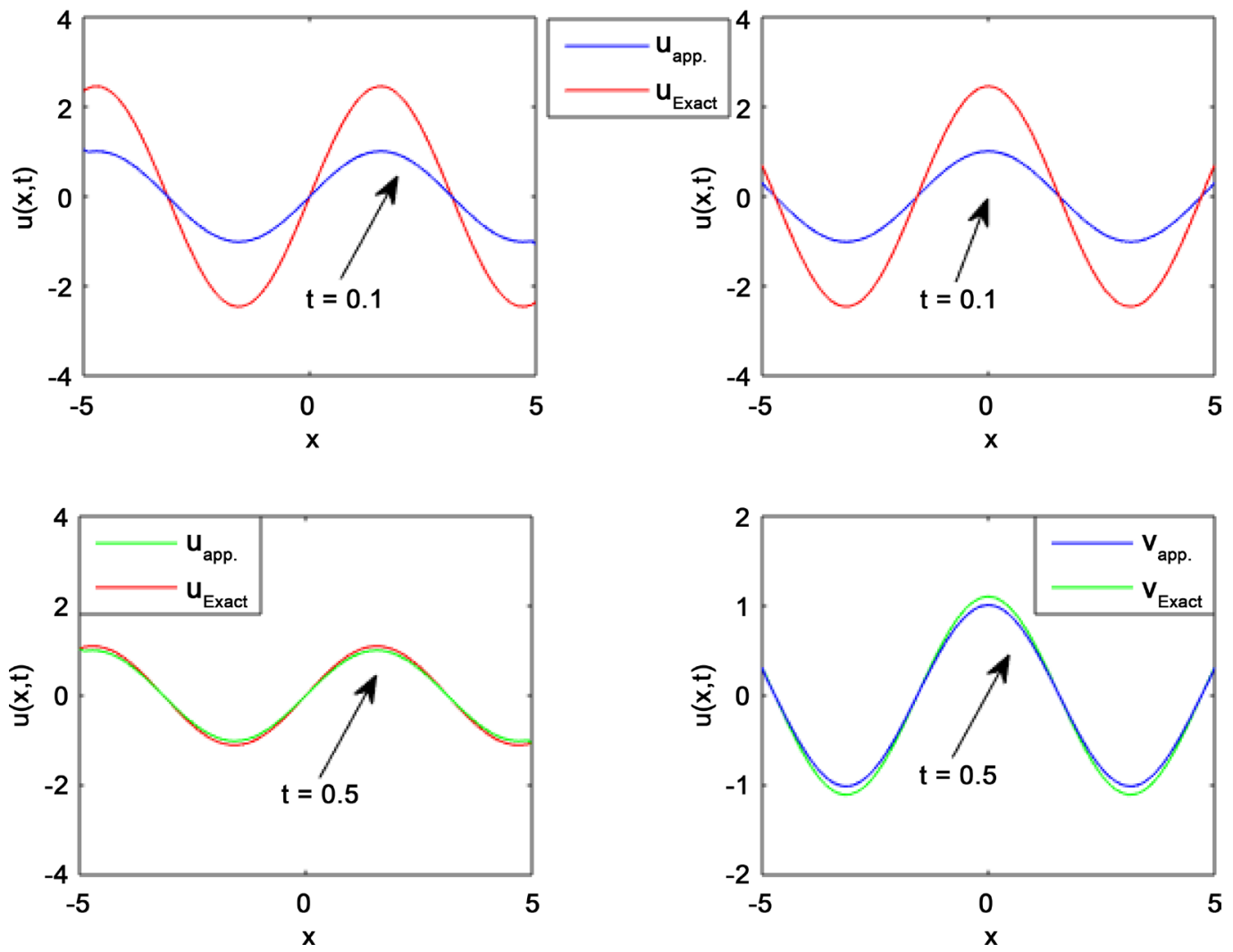

Figure 6. Results for FTCS scheme on Coupled Linear System. 

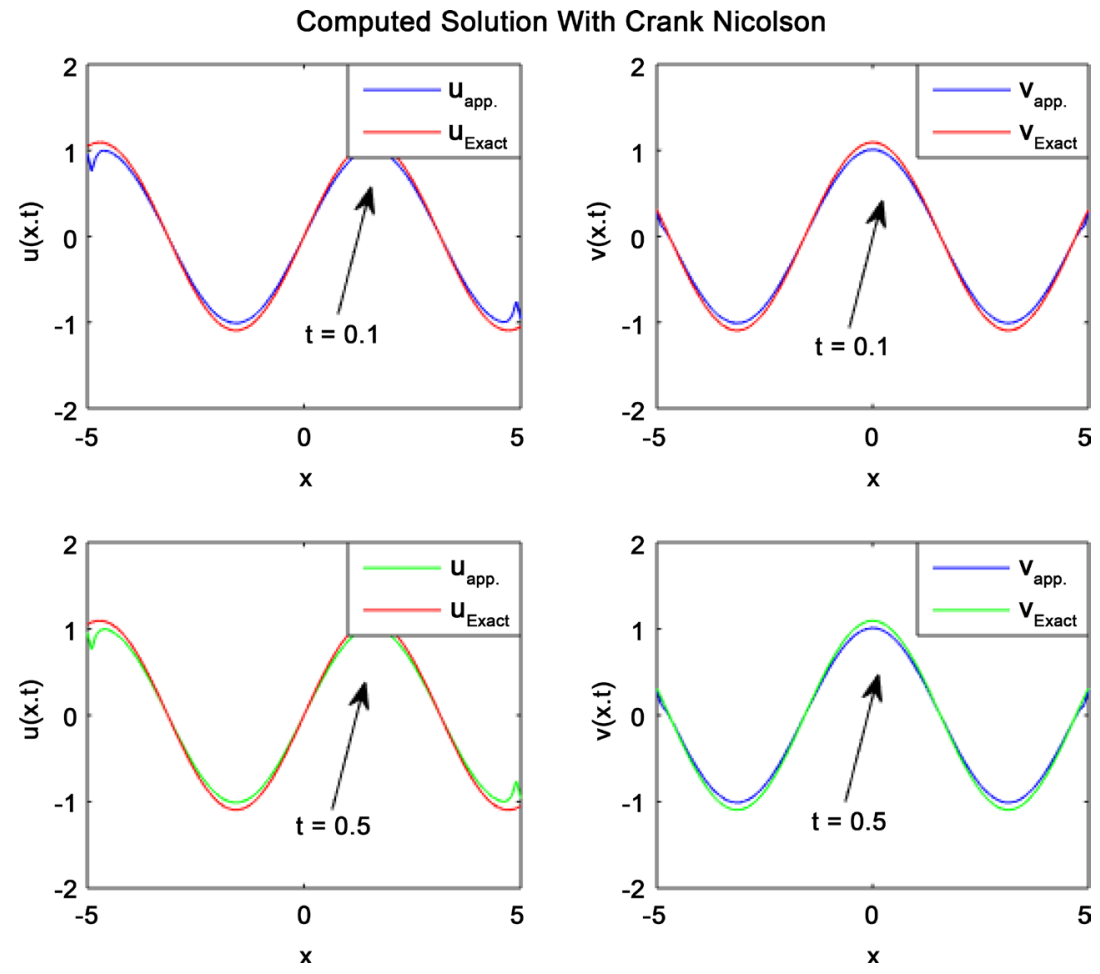

Figure 7. Results for Douglas scheme and error profile with common region.

Computed Solution With Crank Nilcolson
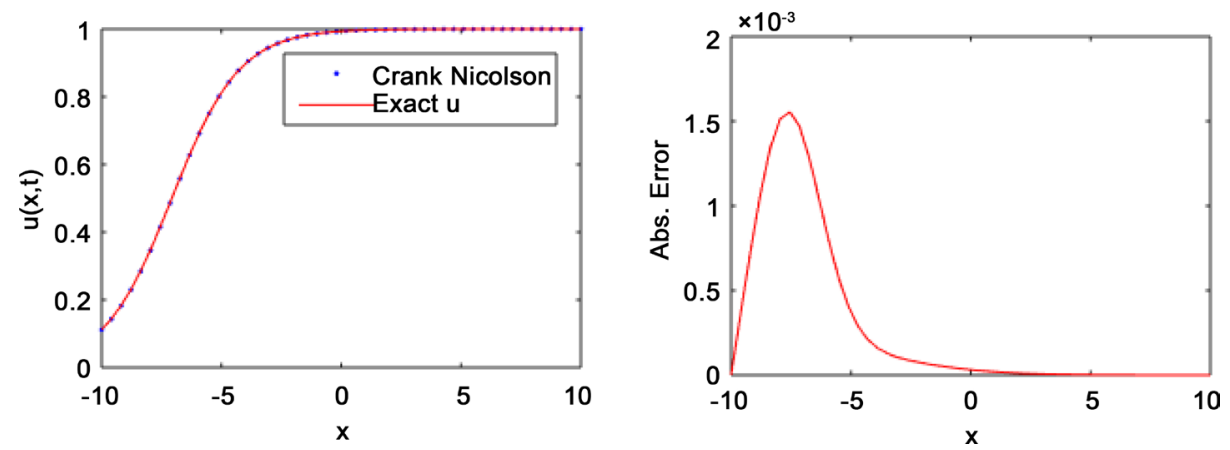

Figure 8. Results for Crank Nicolson scheme and error profile on Non-linear Generalized Fisher's equation.

difference schemes. In many cases, numerical experimentation, such as solving the finite difference schemes using progressively smaller grid spacing and examining the behaviour of the sequence of the values of $u(x, t)$ obtained at given points, is the suitable method available with which to assess the numerical model. The various methods of obtaining a finite difference numerical model corresponding to a particular mathematical model may result in either explicit or implicit finite difference schemes. Explicit schemes are conditionally stable and implicit schemes are unconditionally stable. Two implicit schemes are also applied to improve accuracy, stability restrictions and consistency in solution. It can be observed that the computed results show excellent agreement with the analytical solution. Our main purpose of this research is to improve accuracy in result. Accuracy in results is glanced from figures and tables. 
Computed Solution With Douglas
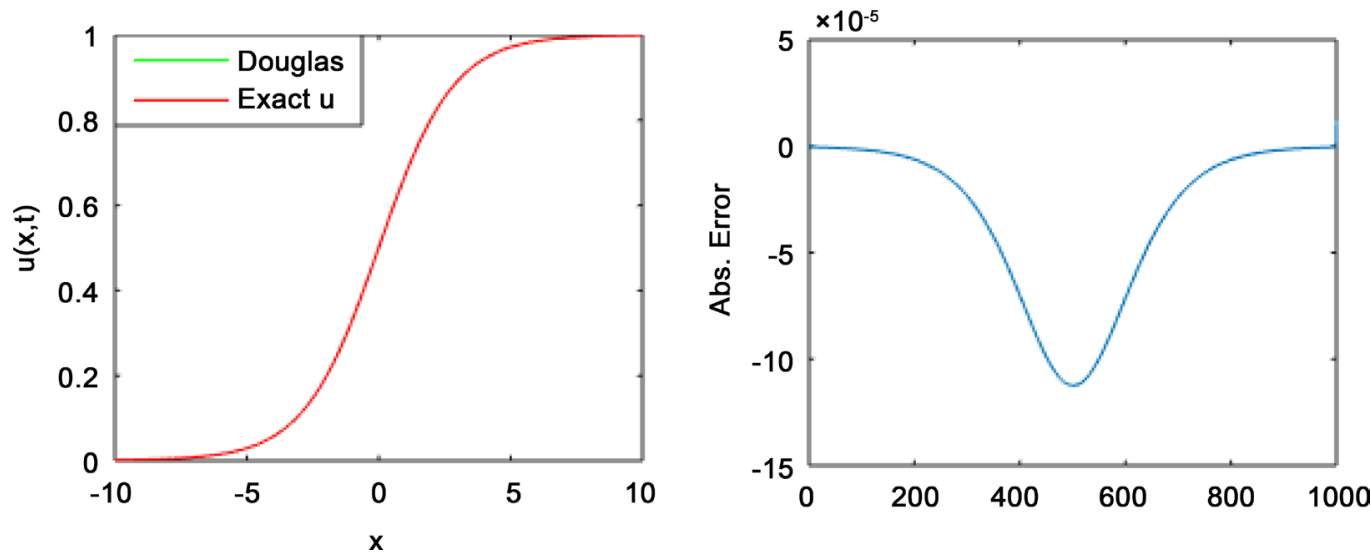

Figure 9. Results for Douglas scheme and error profile on Non-linear Generalized Fisher's equation.

\section{Acknowledgements}

Bader Saad Alshammari and Prof. Daoud Mashat are very thankful to Dr Muhammad Faheem Afzaal, Department of Chemical Engineering, Imperial College London and Vineet K. Srivastava, Scientist, ISTRAC/ISRO, Bangalore, India for thoughtful remarks. This research was supported by Department of Mathematics, division of Numerical Analysis, King Abdulaziz University, Jeddah, Saudi Arabia.

\section{Conflict of Interest}

There is no conflict of interest in this research paper.

\section{References}

[1] Kristiansen, K.K. (2008) Reaction Diffusion Models in Mathematical Biology. Technical University of Denmark, the Department of Mathematics, Lyngby.

[2] Cosner, C.C. (2014) Reaction Diffusion Equations and Ecological Modelling. Department of Mathematics, University of Miami, Coral Gables.

[3] Li, J.L. and Chen, Y.T. (2008) Computational Partial Differential Equations Using MATLAB. University of Nevada, Las Vegas, Paradise, 79-120.

[4] Abdullaev, U.G. (1994) Stability of Symmetric Travelling Waves in the Cauchy Problem for the KPP Equation. Differential Equations, 30, 377-386.

[5] Logan, D.J. (1984) An Introduction to Nonlinear Partial Differential Equations. John Wiley \& Sons, New York.

[6] Canosa, J.C. (1973) On a Nonlinear Diffusion Equation Describing Population Growth. IBM Journal of Research Development, 17, 307-313. https://doi.org/10.1147/rd.174.0307

[7] Gazdag, J.G. and Canosa, J.C. (1974) Numerical Solutions of Fisher's Equation. Journal of Applied Probability, 11, 445-457. https://doi.org/10.1017/S0021900200096236

[8] Tang, S.T., Qin, S.Q. and Weber, R.O. (1991) Numerical Solution of a Non Linear Reaction Diffusion Equation. Chinese J. of Appl. Math, and Mech., 12, 703-709. 
[9] Tang, S.T. and Weber, R.O. (1991) Numerical Study of Fisher's Equation by a Petrov-Galerkin Finite Element Method. Journal of the Australian Mathematical Society Series B, 33, 27-38. https://doi.org/10.1017/S0334270000008602

[10] Khaled, K.A. (2001) Numerical Study of Fisher's Diffusion Reaction Equation by the Sinc Collocation Method. Journal of Computational and Applied Mathematics, 137, 245-255. https://doi.org/10.1016/S0377-0427(01)00356-9

[11] Wang, X.Y. (1988) Exact and Explicit Solitary Wave Solutions for the Generalized Fishers Equation. Physics Letters A, 131, 227-279.

https://doi.org/10.1016/0375-9601(88)90027-8

[12] Dag, I.D., Sahin, A.S. and Korkmaz, A.K. (2010) Numerical Investigation of the Solution of Fishers Equation via the B-Spline Galerkin Method. Numerical Methods for Partial Differential Equations, 26, 1483-1503.

[13] Mittal, R.C. and Rajni, R.R. (2016) A Study of One Dimensional Nonlinear Diffusion Equations by Bernstein Polynomial Based Differential Quadrature Method. Journal of Mathematical Chemistry, 2016, 1-23.

[14] Rahman, K.R., Helil, N.H. and Yimin, A.R. (2010) Some New Semi-Implicit Finite Difference Schemes for Numerical Solution of Burgers Equation. International Conference on Computer Application and System Modeling, 14, 451-455. https://doi.org/10.1109/ICCASM.2010.5622119

[15] Fisher, R.A. (1936) The Wave of Advance of Advantageous Genes. Annals of Eugenics, 7, 355-369. https://doi.org/10.1111/j.1469-1809.1937.tb02153.x

[16] Kolmogorov, A.K., Petrovsky, N.P. and Piscounov, S.P. (1937) Etude de I Equations de la Diffusion avec Croissance de la Quantitate de Matiere et Son Application a un Probolome Biologique. Bulletin University Moscow, 1, 1-25.

[17] Newman, W.I. (1980) Some Exact Solutions to a Non-Linear Diffusion Problem in Population Genetics and Combustion. Journal of Theoretical Biology, 85, 325-334. https://doi.org/10.1016/0022-5193(80)90024-7

[18] Tuckwell, H.C. (1998) Introduction to Theoretical Neurobiology. Cambridge University Press, Cambridge.

[19] Arnold, R.A., Showalter, K. and Tyson, J.J. (1987) Propagation of Chemical Reactions in Space. Journal of Chemical Education, 64, 740-744.

https://doi.org/10.1021/ed064p740

[20] Argyris, J.A., Haase, M.H. and Heinrich, J.C. (1991) Finite Approximation to TwoDimensional Sine Gordon Equations. Computer Methods in Applied Mechanics and Engineering, 86, 1-26. https://doi.org/10.1016/0045-7825(91)90136-T

[21] Grimshaw, R.G. and Tang, S.T. (1990) The Rotation-Modified Kadomtsev Petviashvili Equation: An Analytical and Numerical Study. Studies in Applied Mathematics, 83, 223-248. https://doi.org/10.1002/sapm1990833223

[22] Ames, W.F. (1969) Finite Difference Methods for Partial Differential Equations. Academic Press, New York.

[23] Ablowitz, M.J. and Zeppetella, A.Z. (1979) Explicit Solutions of Fisher's Equation for a Special Wave Speed. Bulletin of Mathematical Biology, 41, 835-840. https://doi.org/10.1007/BF02462380

[24] Kanti, P.K. and Lajja, V.L. (2011) A Note on Crank-Nicolson Scheme for Burgers Equation. Applied Mathematics, 2, 888-899.

[25] Srivastava, V.K. and Tamsir, M.T. (2012) Crank Nicolson Semi Implicit Approach for Numerical Solution of Two Dimensional Coupled Nonlinear Burgers Equations. International Journal of Applied Mechanics and Engineering, 17, 571-581. 
[26] Aronson, D.G. and Weinberger, H.F. (1978) Multidimensional Non-Linear Diffusion Arising in Population Genetics. Advance in Mathematics, 30, 33-76. https://doi.org/10.1016/0001-8708(78)90130-5

[27] Mazumder, S.M. (2015) Numerical Methods for Partial Differential Equations: Finite Difference and Finite Volume Methods. Academic Press, New York.

[28] Williams, S.W. and Chow, P.L. (1978) Nonlinear Reaction-Diffusion Models for Interacting Populations. Journal of Mathematical Analysis and Applications, 62, 157-169. https://doi.org/10.1016/0022-247X(78)90227-5

[29] Whitham, G.B. (1974) Linear and Nonlinear Waves. John Wiley Sons, New York.

[30] Babuska, I.B. (1968) Numerical Stability in Mathematical Analysis. IFIP Congress, Amsterdam, 11-23.

Submit or recommend next manuscript to SCIRP and we will provide best service for you:

Accepting pre-submission inquiries through Email, Facebook, LinkedIn, Twitter, etc. A wide selection of journals (inclusive of 9 subjects, more than 200 journals)

Providing 24-hour high-quality service

User-friendly online submission system

Fair and swift peer-review system

Efficient typesetting and proofreading procedure

Display of the result of downloads and visits, as well as the number of cited articles

Maximum dissemination of your research work

Submit your manuscript at: http://papersubmission.scirp.org/

Or contact am@scirp.org 